\title{
Allelic effects on starch structure and properties of six starch biosynthetic genes in a rice recombinant inbred line population
}

\author{
Jixun Luo ${ }^{1,2}$, Stephen A Jobling ${ }^{1}$, Anthony Millar ${ }^{2}$, Matthew K Morell ${ }^{1,3}$ and Zhongyi Li ${ }^{* *}$
}

\begin{abstract}
Background: The genetic diversity of six starch biosynthetic genes (WX, SSI, SSIIa, SBEI, SBEIla and SBEIII) in indica and japonica rices opens an opportunity to produce a new variety with more favourable grain starch quality. However, there is limited information about the effects of these six gene allele combinations on starch structure and properties. A recombinant inbred line population from a cross between indica and japonica varieties offers opportunities to combine specific alleles of the six genes.
\end{abstract}

Results: The allelic (indica vs japonica) effects of six starch biosynthetic genes on starch structure, functional properties, and abundance of granule bound proteins in rice grains were investigated in a common genetic background using a recombinant inbred line population. The indica Wx (WXi) allele played a major role while indica SSI (SSII), japonica SSIla (SSIIaj) and indica SBEI (SBEII) alleles had minor roles on the increase of amylose content. SSIlaj and japonica SBEIllb (SBEllbj) alleles had a major and a minor role on high ratio of $\sum D P \leq 10$ to $\sum D P \leq 24$ fractions ( $R_{C L 10 / 24}$ ), respectively. Both major alleles (Wxi and SSIIaj) reduced peak viscosity (PV), onset, peak and end gelatinization temperatures (GTs) of amylopectin, and increased amylose-lipid complex dissociation enthalpy compared with their counterpart-alleles, respectively. SBEllai and SBEIlbj decreased PV, whereas SSli and SBEIllbj decreased FV. SBEli reduced setback viscosity and gelatinization enthalpy. $R_{C L 10 / 24}$ of chain length distribution in amylopectin is negatively correlated with PV and BD of paste property and GTs of thermal properties. We also report RILs with superior starch properties combining Wxi, SSlj, SSIlaj, SBEli and SBEIlbj alleles. Additionally, a clear relation is drawn to starch biosynthetic gene alleles, starch structure, properties, and abundance of granule bound starch biosynthetic enzymes inside starch granules.

Conclusions: Rice Wxi and SSIlaj alleles play major roles, while SSli, SBEli, SBEllai and SBEIllbj alleles have minor roles in the determination of starch properties between indica and japonica rice through starch structural modification. The combination of these alleles is a key factor for starch quality improvement in rice breeding programs. $R_{C L 10 / 24}$ value is critical for starch structure and property determination.

Keywords: Rice; Starch; Starch synthases; Starch branching enzymes; Starch property; RVA; DSC

\section{Background}

Rice is the most important cereal crop in developing countries. It feeds over half of the world's population, which is critical to sustain population growth (Toriyama et al. 2005). In rice grains starch is the major component that primarily controls rice quality (Umemoto et al. 2008). There are two major rice subspecies, indica and japonica. The diversity of starch properties between indica and

\footnotetext{
* Correspondence: zhongyili@csiro.au

'CSIRO Agriculture Flagship, GPO Box 1600, Canberra ACT 2601, Australia

Full list of author information is available at the end of the article
}

japonica varieties have been widely studied, and the alleles of genes that affect the traits have been found through a number of approaches (Han et al. 2004; Tian et al. 2009, 2010; Kharabian-Masouleh et al. 2011, 2012; Zhao et al. 2011). In terms of rice traits, cooking and sensory properties are the major determinants for consumers' acceptance of a rice variety. In the absence of a clear knowledge of sensory properties, amylose content, gelatinization temperature and gel consistency of rice flour have been widely used as important indicators of quality for rice varietal development (Cuevas and Fitzgerald 2012). 
Amylose content (AC) of rice starch, affecting the cooking and eating properties, is determined by the activity of granule-bound starch synthase I (GBSSI) encoded by the $W x$ gene (Wang et al. 1995; Umemoto and Terashima 2002). A number of alleles were reported to be associated with starch AC variation. However, $W x^{a}$ and $W x^{b}$ dominate the functional alleles among indica and japonica subspecies, with high and intermediate GBSSI protein production, respectively (Sano 1984; Wang et al. 1995). They can be distinguished mainly by a G/T single nucleotide polymorphism (SNP) at $5^{\prime}$ splicing site of the first intron (Hirano et al. 1998) that resulted in differential splicing of the first intron. Gel consistency in rice is also related to AC (Tan \& Corke 2002; Septiningsih et al. 2003; Zheng et al. 2007) and its quantitative trait locus mapped to the $W x$ locus has been reported (He et al. 1999; Lanceras et al. 2000).

Gelatinization temperature of rice starch is mainly determined by starch synthase IIa (SSIIa) (Govindaraj et al. 2009; Cuevas et al. 2010; Gao et al. 2011). Allelic effects of SSIIa in rice were reported earlier, and up to 9 SNP alleles were identified (Nakamura et al. 2005b; Umemoto and Aoki 2005; Bao et al. 2006; Yu et al. 2010). Two replacements of amino acid residues at the C-terminal region of the SSIIa protein were found to affect SSIIa activity and amylopectin structure and properties in grains (Nakamura et al. 2005b).

Aside from Wx and SSIIa, other starch synthetic genes are also involved in the modification of amylopectin structure and starch properties in rice varieties. The expression of SSI gene in indica rice is lower than that of japonica rice which reduces the synthesis of short chains in indica rice amylopectin (Takemoto-Kuno et al. 2006). A mutation in the SBEI gene leads to a decrease in long chains ( $\mathrm{DP} \geq 37)$ and intermediate chains (DP 12 to 21$)$, increases in short chains $(\mathrm{DP} \leq 10)$ and intermediate chains (DP 24 to 34) and a reduction in the onset, peak and end gelatinization temperature (Xie et al. 1999; Satoh et al. 2003). For the SBEIIb gene, a SNP was determined by Han et al. (2004) in the 3'UTR, between indica and japonica varieties of rice. Taking advantages of nucleotide polymorphisms, genetic markers have been developed for genotyping a series of starch biosynthetic genes to improve grain starch quality in rice breeding programs (Han et al. 2004; Yan et al. 2007; Tian et al. 2010). Nonetheless, nearly all these studies are carried out in isolated individual breeding lines with diverse genetic backgrounds.

The starch properties are highly related to the composition and chemical structure of starch not only in rice but also other cereal grains. Two types of glucan polysaccharides, amylose and amylopectin, comprise starch. The former molecule (approximately $20 \sim 30 \%$ of starch) is a long linear glucose polymer ( $1 \%$ branched points) which is synthesised by GBSSI, through adding ADP- glucose to existing $\alpha-(1-4)$ glucan chains (Nelson and Rines 1962; Tsai 1974; Fedoroff et al. 1983; Shure et al. 1983). The latter (approximately $70 \sim 80 \%$ of starch) is a much larger molecule with frequent $\alpha-(1-6)$ branches formed by multiple enzymes, for example, starch synthases (SSs) and starch branching enzymes (SBEs) (Tetlow et al. 2004; Hannah and James 2008). The isoforms of SSs and SBEs differ in their activities in amylopectin synthesis in a developing endosperm. SSI elongates very short glucan chains to a degree of polymerisation (DP) 10 (Commuri and Keeling 2001; Fujita et al. 2006), while SSIIa is endosperm specific and is involved in the polymerization of intermediate chains (DP $10 \sim 24$ ) by elongating short chains (DP $<10)$ of amylopectin (Nakamura et al. 2005b). The formation of $\alpha-(1-6)$ linkage in amylopectin molecules is mainly performed by SBEI and SBEII (Gao et al. 1996). SBEI transfers glucan chains with a wide range of $\mathrm{DP} \leq 35$, while SBEIIa is involved in the transferring of chains with DP $6 \sim 15$ and SBEIIb forms almost only short chains of DP 6 and 7 (Mizuno et al. 2001; Nishi et al. 2001; Nakamura et al. 2010). The activities of these enzymes potentially affect starch structure and properties.

In this study, the polymorphic effects of six starch biosynthetic genes (Wx, SSI, SSIIa, SBEI, SBEIIa and SBEIIb) on starch structure and properties were studied in a common genetic background, using a recombinant inbred line (RIL) population derived from crossing IR64 (indica, $i$ ) and Nipponbare (japonica, $j$ ). With two alleles ( $i$ or $j$ ) for six different genes there are 64 potential combinations theoretically. In this work we have compared $i$ or $j$ alleles for each gene in a common background, with 12 combinations representing 64 as a whole. Different approaches such as amylose content assay using size-exclusion chromatography, chain length determination by capillary electrophoresis (CE), rapid viscosity analysis (RVA), and differential scanning calorimetry (DSC) were performed to analyse the starch structure and properties of these samples. Starch granule bound proteins (GBPs) were analysed to study the relation between those proteins and starch structure and properties.

\section{Results}

\section{Grouping of recombinant inbred lines}

Six allele groups were selected from recombinant inbred lines corresponding to the 6 major starch synthetic enzyme genes, and parental lines (IR64 and Nipponbare) differing in their alleles ( $i$ vs $j$ ) for each of the 6 genes. Thus we segregated the RILs into six allele groups according to the allele combinations (Figure 1) to analyse the effects of $i$ vs $j$ alleles of different starch biosynthetic genes on starch structure and properties. The allele types of these six genes of each RIL are described in Table 1, and are designated as follows: line 3-14-12 and 3-14-13 as Wxi and Wxj; line 3-5-15-11 and 3-6-20 as 


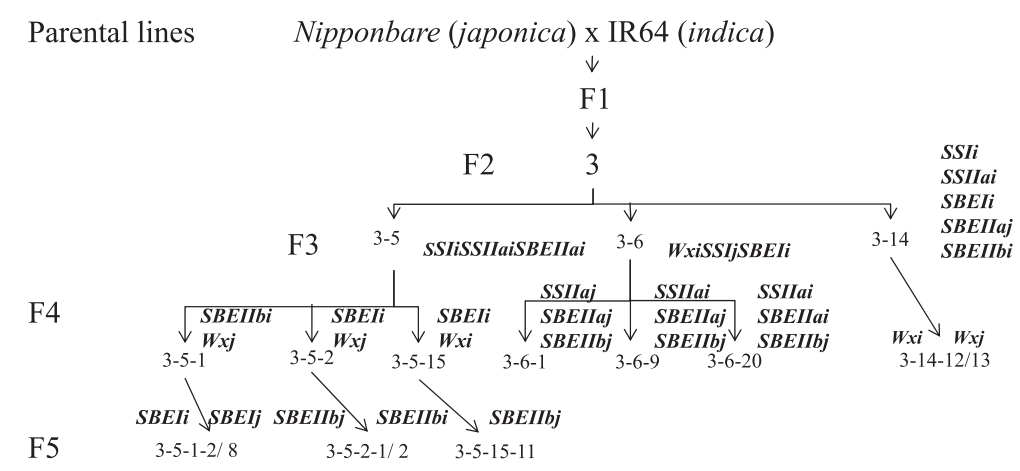

Figure 1 Generation of RILs for each of two alleles of six starch synthetic genes. Six genes for genotyping of alleles are WX, SSI, SSIla, SBEI, SBEIla, SBEIllb. Parental lines are IR64 and Nipponbare. The arrows indicate descendants of each line. The numbers with slash line indicate the name of each line. The numbers following ' $F$ ' imply the generation on the left of the figure. The italic letters followed by ' $i$ ' or ' $j$ ' (indicating indica and japonica, respectively) indicate alleles of the RILs.

SSI $i$ and SSIj; line 3-6-9 and 3-6-1 as SSIIai and SSIIaj; line 3-5-1-2 and 3-5-1-8 as SBEIi and SBEIj; line 3-6-20 and 3-6-9 as SBEIIai and SBEIIaj; and line 3-5-2-2 and 3-5-2-1 as SBEIIbi and SBEIIbj. Meanwhile, we also separated RILs in $W x i$ and $W x j$ allele groups in some of the following analyses, because $W x$ alleles were well known in determining various starch characteristics.

\section{Amylose content}

Although the $W x i$ allele played a major role in the determination of increased AC, other alleles also showed impacts on AC. The range of AC of selected RILs was $11 \sim 35 \%$ approximately of rice grain starch (Figure 2). Six allele groups can be classified into two AC groups depending on the $W x$ allele. The $W x i$ allele group contained $17.7 \sim 35.3 \%$ amylose in starch (including Wxi allele and SSI, SSIIa and SBEIIa groups), whereas $W x j$ allele group contained $11.1 \sim 18.4 \%$ (including $W x j$ allele and SBEI and SBEIIb groups), with parental lines IR64 (21.2\%) and Nipponbare (11.8\%) fitting in their respective $W x$ groups. Between the Wxi allele group (containing average $22.0 \%$ amylose) and the $W x j$ allele group (containing average $13.1 \%$ amylose), the former contained $~ 9 \%$ significantly higher AC than the latter. Within Wxi allele group, starch samples from Wxi allele lines contained significantly higher AC (35.3\%) than all other allele groups, and starch from SSIi $(22.8 \%)$ and SSIIaj (20.9\%) alleles contained higher AC than other four alleles (SSIj, SSIIai, SBEIIai and SBEIIaj) (Figure 2). Within $W x j$ allele group, SBEIi allele lines (18.4\%) contained significantly higher $\mathrm{AC}$ than those from other four allele groups, among which $\mathrm{AC}$ was not significantly different.

\section{Chain length distribution (CLD) of debranched starch}

In this study, linear glucan chains of DP $\leq 24$ comprised up to $80 \%$ mole of molecules in amylopectin of rice grains (Table 2). SSIIai played a major role, while SBEIIbi played a minor role in accumulating intermediate chains (DP12 22). The differences in \% normalised distribution were obtained by subtracting the CLD of japonica allele from indica allele in each gene group, respectively (Figure 3 ). The profound difference in CLD was obtained in SSIIa allele group (Figure 3C). SSIIai allele starch contained fewer short chains at DP6 DP11, more intermediate chains at DP12 22 and long chains at DP30 45 compared with that of SSIIaj allele starch. Limited variations were found in the other five gene groups, however, SBEIIbi allele showed up to $0.5 \%$ normalised

Table 1 Genotypes of RILs of six starch biosynthetic enzyme groups

\begin{tabular}{|c|c|c|c|c|c|c|c|c|c|c|c|c|}
\hline Genotype & $W x i$ & $W x j$ & SSli & SSIj & SSIllai & SSIIaj & SBEli & SBElj & SBEIlai & SBEIlaj & SBEIlbi & SBEIlbj \\
\hline Lines & $3-14-12$ & $3-14-13$ & $3-5-15-11$ & $3-6-20$ & $3-6-9$ & $3-6-1$ & $3-5-1-2$ & $3-5-1-8$ & $3-6-20$ & $3-6-9$ & $3-5-2-2$ & $3-5-2-1$ \\
\hline$W x$ & $\mathrm{i}$ & j & i & & $\mathrm{i}$ & & j & & i & & j & \\
\hline SSI & i & & i & j & j & & i & & j & & i & \\
\hline SSIIa & i & & i & & i & j & i & & i & & $\mathrm{i}$ & \\
\hline SBEI & i & & i & & i & & i & j & i & & i & \\
\hline SBEIla & j & & i & & j & & i & & i & j & i & \\
\hline SBEIIb & i & & j & & j & & i & & j & & i & j \\
\hline
\end{tabular}

Names of each RIL are shown in the first row of corresponding column. 'i' and ' $j$ ' are short abbreviation for indica and japonica genotypes, indicating their parental origins, respectively. The second row of each column indicates the corresponding RILs. The names of six starch biosynthetic genes are listed in the first column. 


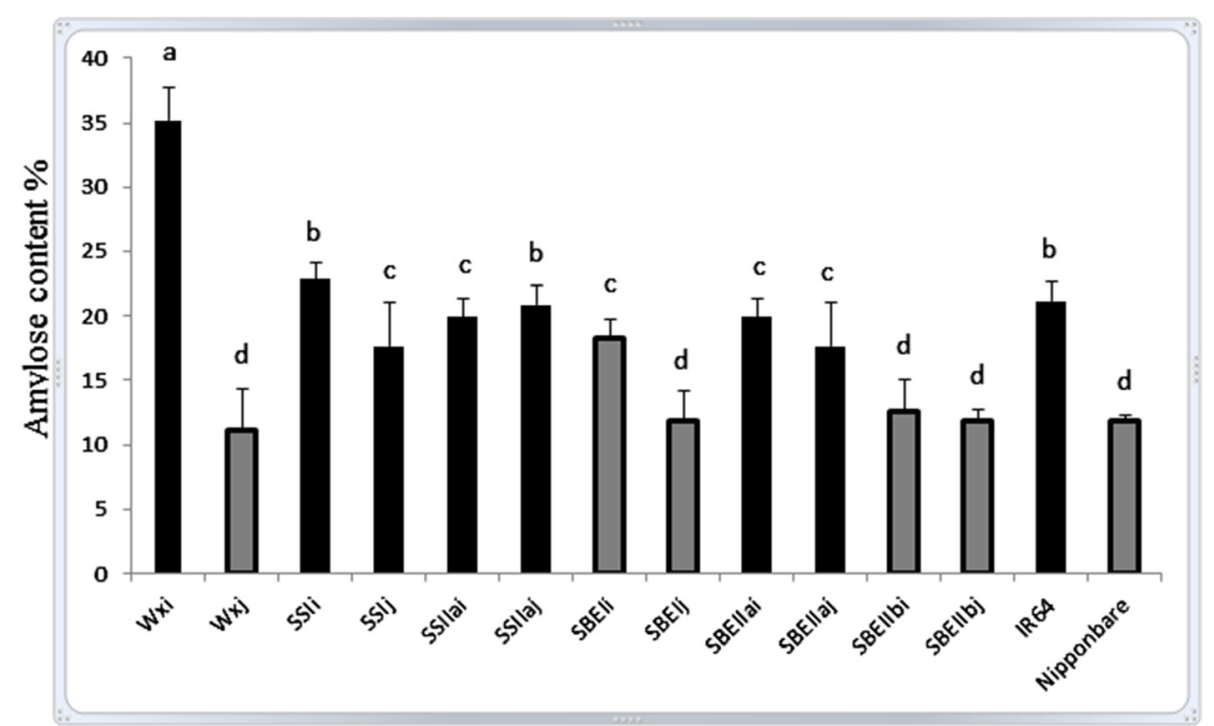

Figure 2 Comparison of amylose content among 12 alleles of six starch synthetic genes using the SEC method. Starches from five plants of each allele were isolated and analysed seperately. Two replicates were set up for each sample. The columns indicate starch AC of RIL grains. Black columns indicate those allelles containing $W x i$ allele and grey columns indicate those allelles containing $W x j$ allele. The error bars show the standard error of the mean. The identity of each column is indicated underneath. Columns with different letters are significantly different at $p<0.05$.

distribution of fewer chains at DP10 13 than SBEIIbj allele (Figure 3F).

Based on Nakamura's study (Nakamura et al. 2002), indica and japonica type amylopectin from the majority of cultivated Asian rice strains can be distinguished by

Table 2 Chain length fractions of RILs of six starch biosynthetic enzyme allele groups

\begin{tabular}{|c|c|c|c|c|}
\hline Genotype & $\Sigma \mathrm{DP} \leq 10 \%$ & $\Sigma \mathrm{DP} \leq 24 \%$ & $\Sigma D P>24 \%$ & $\mathrm{R}_{\mathrm{CL} 10 / 24} \times 10^{-2}$ \\
\hline Wxi & $10.3 \pm 0.1^{e}$ & $78.4 \pm 0.1^{b}$ & $21.7 \pm 0.3^{a}$ & $13.1 \pm 0.1^{e}$ \\
\hline$W x j$ & $10.0 \pm 0.1^{\mathrm{e}}$ & $78.4 \pm 0.1^{b}$ & $21.6 \pm 0.4^{\mathrm{a}}$ & $12.7 \pm 0.1^{\mathrm{e}}$ \\
\hline SSli & $10.8 \pm 0.1^{d}$ & $78.8 \pm 0.1^{b}$ & $21.2 \pm 0.2^{b}$ & $13.6 \pm 0.1^{d}$ \\
\hline SSlj & $10.8 \pm 0.1^{d}$ & $79.6 \pm 0.1^{a}$ & $20.5 \pm 0.2^{b}$ & $13.5 \pm 0.1^{d}$ \\
\hline SSIllai & $10.6 \pm 0.1^{d}$ & $78.3 \pm 0.1^{b}$ & $21.7 \pm 0.2^{b}$ & $13.5 \pm 0.1^{d}$ \\
\hline SSIIIaj & $15.9 \pm 0.1^{b}$ & $76.7 \pm 0.1^{c}$ & $23.3 \pm 0.4^{a}$ & $20.7 \pm 0.1^{b}$ \\
\hline SBEli & $9.8 \pm 0.1^{\mathrm{e}}$ & $79.3 \pm 0.1^{a}$ & $20.7 \pm 0.5^{b}$ & $12.4 \pm 0.1^{f}$ \\
\hline SBElj & $9.5 \pm 0.1^{f}$ & $77.7 \pm 0.1^{b}$ & $22.4 \pm 0.3^{a}$ & $12.2 \pm 0.1^{f}$ \\
\hline SBEllai & $10.8 \pm 0.1^{d}$ & $79.6 \pm 0.1^{a}$ & $20.5 \pm 0.2^{b}$ & $13.5 \pm 0.1^{d}$ \\
\hline SBEllaj & $10.6 \pm 0.1^{d}$ & $78.3 \pm 0.1^{b}$ & $21.7 \pm 0.2^{b}$ & $13.5 \pm 0.1^{d}$ \\
\hline SBEIlbi & $10.2 \pm 0.2^{e}$ & $78.5 \pm 0.2^{b}$ & $21.5 \pm 0.8^{\mathrm{a}}$ & $12.9 \pm 0.2^{\mathrm{e}}$ \\
\hline SBEIlbj & $11.1 \pm 0.3^{d}$ & $81.2 \pm 0.3^{a}$ & $18.8 \pm 1.2^{c}$ & $13.7 \pm 0.2^{d}$ \\
\hline \multicolumn{5}{|c|}{ Parent control } \\
\hline IR64 & $12.8 \pm 0.1^{c}$ & $79.7 \pm 0.2^{a}$ & $19.3 \pm 0.3^{b}$ & $16.1 \pm 0.1^{c}$ \\
\hline Nipponbare & $22.9 \pm 0.0^{a}$ & $80.1 \pm 0.1^{a}$ & $19.9 \pm 0.2^{b}$ & $28.6 \pm 0.1^{a}$ \\
\hline
\end{tabular}

Values are mean values of percentages of the mole basis of five different RIL lines of each allele with two replicates. $R_{C L 10 / 24}$ is the ratio of $\sum D P \leq 10 / \Sigma D P \leq 24$. IR64 and Nipponbare are the parental lines. Values with different characters are significantly different at $p<0.01$. the ratio of $\Sigma \mathrm{DP} \leq 10$ to $\Sigma \mathrm{DP} \leq 24$ fractions $\left(\mathrm{R}_{\mathrm{CL10} / 24}\right)$ of CLD. Our present study showed the $R_{\mathrm{CL} 10 / 24}$ value was 0.16 for IR64 and 0.29 for Nipponbare (Table 2), and most of the selected RILs were smaller than 0.14 , suggesting that they were indica type. With the only exception of SSIIaj allele, its $\mathrm{R}_{\mathrm{CL} 10 / 24}$ value was slightly over 0.20 . The highest value of SSIIaj resulted from a high percentage of short chain fraction $\left(\sum D P \leq 10\right.$, significantly higher than that of SSIIai allele, and $43 \sim$ $67 \%$ higher than the other RILs) and low percentage of short and intermediate chain fraction ( $\Sigma D P \leq 24$, statistically significantly lower than that of SSIIai allele) in the starch. In terms of the longer chain fraction ( DP > 24), SSIIaj was the highest but not significantly different to a few other alleles in the group (Table 2). Besides SSIIaj, SBEIIbj allele was also detected with more short chains in starch than the counterpart-allele SBEIIbi.

\section{Starch paste viscosity}

By analysing RVA characteristics pasting properties of starch were determined as reported in previous studies (Sasaki et al. 2000; Han and Hamaker 2001; Chen et al. 2003). The RVA characteristics involved in this study were peak viscosity (PV), trough (Tr), breakdown (BD), final viscosity (FV), setback (SB) and peak time (PT). The values of these characteristics were statistically analysed and shown in Table 3. The RVA result revealed that $i$ and $j$ alleles from each of six allele groups 


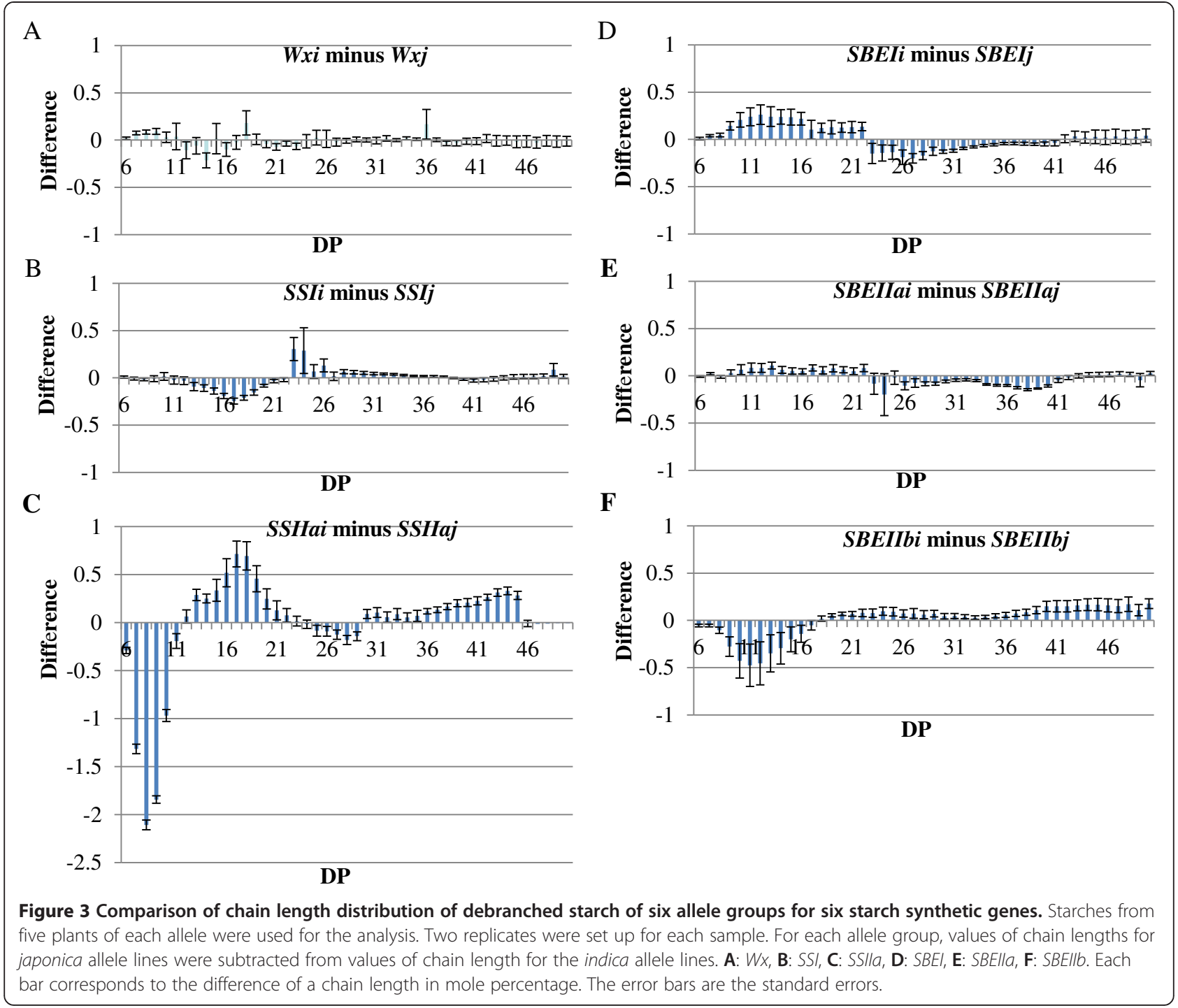

contributed to parameters of starch paste viscosity properties differentially.

IR64 and Nipponbare showed significant differences in all parameters except for FV (Table 3). RILs in Wx, SSIIa, SBEIIa and SBEIIb allele groups had significantly distinct PV between $i$ and $j$ alleles, respectively. PV values of $W x j$ and SSIIai alleles were $\sim 60$ RVU higher than $W x i$ and SSIIaj alleles, whereas the increase in SBEIIaj and SBEIIbi alleles was smaller (31 35 RVU) comparing to SBEIIai and SBEIIbj alleles. Tr was the least diverse RVA parameter in this analysis, showing only about 30 and 10 RVU significant increase in SSIj and SBEIIbi alleles comparing to their counterpartalleles, respectively. Significant differences in BD were obtained in Wx, SSI, SSIIa and SBEIIa allele groups, with the greatest ( $\sim 70$ RVU) between SSIIai and SSIIaj alleles. Compared to their counterparts, the values of BD were increased by $\sim 40$ RVU in $W x j$ allele, and $\sim 30$ RVU in SSI $i$ and SBEIIaj alleles. In terms of FV, significant variations were found in $W x, S S I$ and SBEIIb allele groups, with the highest ( $\sim 0 \mathrm{RVU}$ ) between SSI $i$ and SSIj alleles, $\sim 30$ and 15 RVU in $W x$ and SBEIIb allele groups, respectively. The significant variations of SB were 42 RVU in $W x$ group and 28 RVU in SSIIa group. Less than 10 RVU increase of SB in both SSIj and SBEIj alleles were also significantly different. Significant differences in PT were determined between SSI alleles (0.6 min) and $W x$ group $(0.2 \mathrm{~min})$. The PT values were mainly clustered into the two $W x$ alleles groups, while IR64 was the lowest. Notably, the SSIIaj allele starch exhibited remarked differences in paste viscosities from all the others including parental controls. 
Table 3 Viscosity properties of RIL wholemeal flours analysed using RVA

\begin{tabular}{|c|c|c|c|c|c|c|}
\hline Genotype & Peak viscosity (RVU) & Trough (RVU) & Breakdown (RVU) & Final viscosity (RVU) & Setback (RVU) & Peak time (min) \\
\hline$W x i$ & $227.7 \pm 6.0^{b}$ & $109.5 \pm 8.1^{a}$ & $118.1 \pm 10.1^{b}$ & $243.6 \pm 7.1^{a}$ & $134.0 \pm 1.9^{b}$ & $8.7 \pm 0.08^{a}$ \\
\hline$W x j$ & $283.6 \pm 2.5^{\mathrm{a}}$ & $121.1 \pm 1.2^{\mathrm{a}}$ & $162.4 \pm 3.1^{\mathrm{a}}$ & $213.0 \pm 2.4^{b}$ & $91.9 \pm 1.9^{d}$ & $8.5 \pm 0.02^{b}$ \\
\hline SSli & $210.1 \pm 3.7^{c}$ & $79.1 \pm 3.3^{b}$ & $131.0 \pm 6.2^{b}$ & $194.2 \pm 6.8^{b}$ & $115.2 \pm 3.6^{c}$ & $8.3 \pm 0.12^{b}$ \\
\hline SSlj & $215.6 \pm 6.2^{c}$ & $112.1 \pm 5.1^{\mathrm{a}}$ & $103.6 \pm 1.7^{c}$ & $235.0 \pm 7.9^{a}$ & $122.9 \pm 3.5^{\mathrm{b}}$ & $8.9 \pm 0.02^{a}$ \\
\hline SSIllai & $246.8 \pm 4.2^{b}$ & $109.0 \pm 3.4^{\mathrm{a}}$ & $137.8 \pm 5.0^{\mathrm{b}}$ & $232.5 \pm 5.8^{\mathrm{a}}$ & $123.5 \pm 2.7^{b}$ & $8.8 \pm 0.10^{a}$ \\
\hline SSIIIaj & $181.3 \pm 6.0^{d}$ & $112.9 \pm 7.6^{\mathrm{a}}$ & $68.4 \pm 2.9^{d}$ & $264.4 \pm 12.9^{a}$ & $151.5 \pm 5.4^{a}$ & $8.8 \pm 0.06^{\mathrm{a}}$ \\
\hline SBEli & $252.6 \pm 8.1^{b}$ & $107.9 \pm 2.7^{\mathrm{a}}$ & $144.7 \pm 5.4^{b}$ & $204.4 \pm 3.0^{b}$ & $96.5 \pm 0.4^{d}$ & $8.5 \pm 0.00^{b}$ \\
\hline SBElj & $240.1 \pm 5.0^{b}$ & $118.7 \pm 2.6^{\mathrm{a}}$ & $121.4 \pm 2.4^{\mathrm{b}}$ & $225.1 \pm 3.6^{b}$ & $106.4 \pm 1.5^{c}$ & $8.5 \pm 0.02^{b}$ \\
\hline SBEllai & $215.6 \pm 6.2^{c}$ & $112.1 \pm 5.1^{\mathrm{a}}$ & $103.6 \pm 1.7^{c}$ & $235.0 \pm 7.9^{a}$ & $122.9 \pm 3.5^{b}$ & $8.9 \pm 0.02^{a}$ \\
\hline SBEllaj & $246.8 \pm 4.2^{b}$ & $109.0 \pm 3.4^{\mathrm{a}}$ & $137.8 \pm 5.0^{\mathrm{b}}$ & $232.5 \pm 5.8^{a}$ & $123.5 \pm 2.7^{b}$ & $8.8 \pm 0.10^{\mathrm{a}}$ \\
\hline SBEllbi & $291.8 \pm 2.6^{a}$ & $106.3 \pm 3.4^{\mathrm{a}}$ & $185.6 \pm 5.6^{\mathrm{a}}$ & $200.5 \pm 3.2^{b}$ & $94.3 \pm 2.7^{d}$ & $8.4 \pm 0.04^{b}$ \\
\hline SBEllbj & $256.3 \pm 3.9^{b}$ & $96.7 \pm 4.5^{\mathrm{b}}$ & $159.6 \pm 7.5^{\mathrm{a}}$ & $184.3 \pm 3.6^{c}$ & $87.6 \pm 2.0^{d}$ & $8.4 \pm 0.12^{b}$ \\
\hline \multicolumn{7}{|c|}{ Parent control } \\
\hline IR64 & $267.4 \pm 1.2^{\mathrm{a}}$ & $97.9 \pm 2.3^{\mathrm{a}}$ & $169.5 \pm 3.10^{\mathrm{a}}$ & $216.2 \pm 2.8^{b}$ & $118.3 \pm 1.7^{c}$ & $8.2 \pm 0.08^{c}$ \\
\hline Nipponbare & $214.6 \pm 10.0^{c}$ & $97.0 \pm 4.0^{b}$ & $117.6 \pm 6.05^{b}$ & $195.1 \pm 5.7^{b}$ & $98.1 \pm 1.7^{d}$ & $8.5 \pm 0.02^{b}$ \\
\hline I.s.d. & 29.11 & 23.47 & 28.47 & 32.24 & 14.20 & 0.35 \\
\hline
\end{tabular}

$\mathrm{RVU}$ is rapid viscosity unit. Each value is the mean of 3 biological replicates. Mean values within columns with difference letters are significantly different at $p<0.01$.

\section{Starch thermal properties}

Consistent with previous studies, two primary peaks were observed in DSC curves for rice wholemeal samples: the first one was the initial gelatinization peak at $65 \sim 85^{\circ} \mathrm{C}$ of amylopectin; the second one at around $105^{\circ} \mathrm{C}$ was the amylose-lipid complex dissociation peak (Biliaderis et al. 1985). Onset, peak and end temperatures of the first peak were named as ${ }^{1} \mathrm{To},{ }^{1} \mathrm{Tp}$ and ${ }^{1} \mathrm{Te}$, and those of the second peak as ${ }^{2} \mathrm{To},{ }^{2} \mathrm{Tp},{ }^{2} \mathrm{Te} .{ }^{1} \Delta \mathrm{H}$ was named for the amylopectin gelatinization enthalpy, and ${ }^{2} \Delta \mathrm{H}$ for the dissociation enthalpy of amylose-lipid complexes. As shown in Table 4, the DSC result indicated that SSIIaj allele had a major effect, while $W x i$ allele played a minor role in decreasing ${ }^{1} \mathrm{Tp}$. However, SSIj, SSIIaj and Wxi alleles increased ${ }^{2} \Delta \mathrm{H}$.

Regarding amylopectin gelatinization temperatures, IR64 had significantly higher values in ${ }^{1} \mathrm{To},{ }^{1} \mathrm{Tp}$ and ${ }^{1}$ Te than Nipponbare, whereas the RILs exhibited divergent values. $W x j$ allele group observed $\sim 3^{\circ} \mathrm{C}$ higher ${ }^{1}$ To than those from $W x i$ allele group. There was barely any variation in ${ }^{1}$ To among different alleles within Wxi allele group except SSIIaj allele having $\sim 11^{\circ} \mathrm{C}$ decrease compared with SSIIai allele. In terms of ${ }^{1} \mathrm{Tp}$, $W x j$ allele group overall was $\sim 3^{\circ} \mathrm{C}$ higher than $W x i$ allele group. Within Wxi allele group, SSIIai was $\sim 11^{\circ} \mathrm{C}$ higher than SSIIaj, while SSIj and SBEIIai were $\sim 1$ and $0.5^{\circ} \mathrm{C}$ higher than SSIi and SBEIIaj alleles, respectively. For ${ }^{1} \mathrm{Te}, W x j$ allele group overall was $\sim 2^{\circ} \mathrm{C}$ higher than $W x i$ allele group. There was $\sim 9^{\circ} \mathrm{C}$ increase in SSIIai allele comparing to SSIIaj allele. In terms of ${ }^{1} \Delta \mathrm{H}, W x j$ allele group was $\sim 0.4 \mathrm{~J} / \mathrm{g}$ higher than $W x i$ allele group, except for SBEIi allele $0.3 \mathrm{~J} / \mathrm{g}$ lower than the counterpart-allele.

Regarding dissociation temperatures of amylose-lipid complexes, IR64 wholemeal had significantly higher values of ${ }^{2} \mathrm{To},{ }^{2} \mathrm{Tp}$ and ${ }^{2} \mathrm{Te}$ than Nipponbare. In terms of ${ }^{2}$ To, the only significant variations were observed in SSIIaj and SBEIIai alleles which both had $\sim 2^{\circ} \mathrm{C}$ increase compared with their counterpart-alleles. In terms of ${ }^{2} \Delta \mathrm{H}$, SSIIaj and SSIj alleles were up to $0.5 \mathrm{~J} / \mathrm{g}$ and $0.24 \mathrm{~J} / \mathrm{g}$ higher than SSIIai and SSIi alleles, respectively. Wxi allele group overall exhibited higher energy than Wxj allele group. Within Wxi group, SSIIaj and Wxi were the highestand lowest, whereas in $W x j$ allele group SBEI $i$ allele had higher ${ }^{2} \Delta \mathrm{H}$ than the others. Interestingly, similar to the observation in RVA examination, SSIIaj allele starch also showed remarked differences in thermal properties from others.

\section{Protein analyses in starch granules of mature grains}

The analysis of GBPs prepared from purified starch of mature rice grains showed that four major protein bands with $60 \mathrm{kDa}$ and above were detected in most of the RILs (Figure 4). In parental lines, the top bands at $\sim 88 \mathrm{kDa}$ were identified as SSIIa, $\sim 83 \mathrm{kDa}$ as SBEIIb, $\sim 75 \mathrm{kDa}$ as SSI and $\sim 60 \mathrm{kDa}$ as GBSSI by immunoblotting using specific antibodies (Figure 5).

Comparing the two parental lines, IR64 contained a higher amount of GBSSI, SSI, SSIIa and SBEIIb in the 
Table 4 Thermal characteristics of RIL flours determined using DSC

\begin{tabular}{|c|c|c|c|c|c|c|c|c|}
\hline Genotype & ${ }^{1} \mathrm{To}\left({ }^{\circ} \mathrm{C}\right)$ & ${ }^{1} \mathrm{Tp}\left({ }^{\circ} \mathrm{C}\right)$ & ${ }^{1} \mathrm{Te}\left({ }^{\circ} \mathrm{C}\right)$ & ${ }^{1} \Delta \mathrm{H}(\mathrm{J} / \mathrm{g})$ & ${ }^{2}$ To $\left({ }^{\circ} \mathrm{C}\right)$ & ${ }^{2} \mathrm{Tp}\left({ }^{\circ} \mathrm{C}\right)$ & ${ }^{2} \mathrm{Te}\left({ }^{\circ} \mathrm{C}\right)$ & ${ }^{2} \Delta \mathrm{H}(\mathrm{J} / \mathrm{g})$ \\
\hline$W \times i$ & $73.4 \pm 0.6^{b}$ & $80.1 \pm 0.3^{c}$ & $87.1 \pm 0.6^{c}$ & $3.1 \pm 0.3^{\mathrm{a}}$ & $97.7 \pm 0.4^{a}$ & $104.1 \pm 0.6^{a}$ & $109.1 \pm 1.0^{a}$ & $0.42 \pm 0.08^{c}$ \\
\hline$W x j$ & $76.4 \pm 0.4^{a}$ & $83.0 \pm 0.4^{b}$ & $89.6 \pm 0.0^{a}$ & $3.3 \pm 0.0^{\mathrm{a}}$ & $99.8 \pm 0.5^{a}$ & $103.5 \pm 0.5^{\mathrm{a}}$ & $104.3 \pm 1.8^{\mathrm{a}}$ & $0.21 \pm 0.02^{d}$ \\
\hline SSli & $71.8 \pm 2.5^{\mathrm{b}}$ & $77.6 \pm 0.4^{e}$ & $85.9 \pm 0.7^{d}$ & $2.6 \pm 0.2^{b}$ & $98.9 \pm 1.1^{\mathrm{a}}$ & $102.9 \pm 0.7^{\mathrm{a}}$ & $106.5 \pm 1.5^{\mathrm{a}}$ & $0.36 \pm 0.05^{c}$ \\
\hline SSlj & $73.1 \pm 0.3^{b}$ & $79.3 \pm 0.3^{c}$ & $86.1 \pm 0.5^{c}$ & $2.9 \pm 0.2^{b}$ & $99.4 \pm 1.1^{a}$ & $103.7 \pm 1.3^{\mathrm{a}}$ & $105.7 \pm 2.5^{\mathrm{a}}$ & $0.60 \pm 0.14^{b}$ \\
\hline SSIllai & $71.6 \pm 0.5^{b}$ & $78.7 \pm 0.1^{d}$ & $86.2 \pm 0.3^{c}$ & $2.7 \pm 0.1^{b}$ & $97.3 \pm 0.8^{b}$ & $106.4 \pm 1.3^{\mathrm{a}}$ & $109.6 \pm 1.7^{\mathrm{a}}$ & $0.70 \pm 0.06^{b}$ \\
\hline SSIllaj & $60.5 \pm 0.3^{d}$ & $67.9 \pm 0.5^{9}$ & $77.2 \pm 1.1^{f}$ & $2.8 \pm 0.1^{b}$ & $99.7 \pm 3.8^{\mathrm{a}}$ & $104.2 \pm 4.7^{a}$ & $109.6 \pm 3.6^{a}$ & $1.20 \pm 0.05^{\mathrm{a}}$ \\
\hline SBEli & $76.5 \pm 0.4^{a}$ & $83.4 \pm 0.4^{a}$ & $89.9 \pm 0.4^{a}$ & $2.9 \pm 0.0^{b}$ & $99.0 \pm 1.9^{a}$ & $104.5 \pm 1.2^{\mathrm{a}}$ & $109.5 \pm 0.8^{a}$ & $0.42 \pm 0.01^{c}$ \\
\hline SBElj & $77.4 \pm 0.8^{\mathrm{a}}$ & $84.4 \pm 0.3^{a}$ & $90.9 \pm 0.1^{a}$ & $3.2 \pm 0.1^{\mathrm{a}}$ & $101.0 \pm 0.3^{\mathrm{a}}$ & $105.7 \pm 0.4^{a}$ & $108.0 \pm 1.4^{\mathrm{a}}$ & $0.50 \pm 0.04^{c}$ \\
\hline SBEllai & $73.1 \pm 0.3^{b}$ & $79.3 \pm 0.3^{c}$ & $86.1 \pm 0.5^{c}$ & $2.9 \pm 0.2^{b}$ & $99.4 \pm 1.1^{\mathrm{a}}$ & $103.7 \pm 1.3^{\mathrm{a}}$ & $105.7 \pm 2.5^{\mathrm{a}}$ & $0.60 \pm 0.14^{b}$ \\
\hline SBEllaj & $71.6 \pm 0.5^{b}$ & $78.7 \pm 0.1^{d}$ & $86.2 \pm 0.3^{c}$ & $2.7 \pm 0.1^{\mathrm{b}}$ & $97.3 \pm 0.8^{b}$ & $106.4 \pm 1.3^{\mathrm{a}}$ & $109.6 \pm 1.7^{\mathrm{a}}$ & $0.70 \pm 0.06^{b}$ \\
\hline SBEIlbi & $76.9 \pm 1.0^{a}$ & $83.4 \pm 0.6^{b}$ & $90.4 \pm 0.6^{a}$ & $3.5 \pm 0.0^{a}$ & $101.6 \pm 1.4^{a}$ & $105.7 \pm 0.5^{\mathrm{a}}$ & $108.5 \pm 2.1^{a}$ & $0.28 \pm 0.01^{d}$ \\
\hline SBEllbj & $77.3 \pm 0.2^{a}$ & $83.3 \pm 0.3^{b}$ & $89.2 \pm 0.4^{b}$ & $3.2 \pm 0.0^{\mathrm{a}}$ & $98.0 \pm 0.1^{a}$ & $104.1 \pm 0.5^{\mathrm{a}}$ & $107.7 \pm 2.0^{\mathrm{a}}$ & $0.22 \pm 0.03^{d}$ \\
\hline \multicolumn{9}{|c|}{ Parent control } \\
\hline IR64 & $72.9 \pm 0.1^{b}$ & $80.2 \pm 0.1^{c}$ & $87.5 \pm 0.2^{c}$ & $2.7 \pm 0.1^{b}$ & $97.65 \pm 0.94^{a}$ & $102.5 \pm 0.9^{a}$ & $106.9 \pm 0.4^{a}$ & $0.38 \pm 0.04^{c}$ \\
\hline Nipponbare & $65.2 \pm 1.4^{c}$ & $74.9 \pm 0.1^{f}$ & $83.0 \pm 0.1^{e}$ & $2.7 \pm 0.1^{b}$ & $96.97 \pm 0.93^{b}$ & $101.7 \pm 0.4^{b}$ & $101.8 \pm 1.8^{b}$ & $0.34 \pm 0.01^{c}$ \\
\hline I.s.d. & 2.77 & 1.04 & 1.48 & 0.42 & 4.23 & 4.61 & 5.46 & 0.17 \\
\hline
\end{tabular}

${ }^{1} \mathrm{To},{ }^{1} \mathrm{Tp}$ and ${ }^{1} \mathrm{Te}$ are onset, peak and end gelatinization temperatures, and ${ }^{1} \Delta \mathrm{H}$ is the gelatinization enthalpy at the amylopectin gelatinization peak. ${ }^{2} \mathrm{To},{ }^{2} \mathrm{Tp}$, and ${ }^{2} \mathrm{Te}$ are onset, peak and end dissociation temperatures of amylose-lipid complexes, and ${ }^{2} \Delta \mathrm{H}$ is the dissociation enthalpy at the dissociation peak of amylose-lipid complexes. Each value is the mean of three biological replicates. Mean values within the same column with different letters are significantly different at $p<0.01$.

GBPs than Nipponbare, and SSIIa was barely detectable by immunodetection in Nipponbare (Figure 5A). Consistent with parental lines, GBSSI abundance of Wxi allele was significantly higher than that of $W x j$ allele, while the abundance of SSI, SSIIa and SBEIIb in Wxi and $W x j$ alleles remained at same levels, respectively (Figures $4 \mathrm{~A}, 5 \mathrm{~B}$ ). In SSIIa alleles, the amount of SSI, SSIIa and SBEIIb protein was significantly decreased in SSIIaj allele (Figure 5C). Similarly, only faint bands of SSIIa were detected in SSIIaj RILs (Figure 4C), whereas GBSSI remained at the same level. No significant changes were observed in abundance of the four proteins in SDS-PAGE gels of other groups.

\section{Discussion}

In this work, six starch synthetic gene alleles were used for genotyping progenies from one crossing line between indica and japonica rice. We aimed at understanding the roles of those alleles on starch properties of rice grain. The results showed that both amylose content and amylopectin structure were affected by multiple gene alleles, although some of them play major roles, others play minor roles. However, as matter of time, the roles of other genes involved in starch biosynthesis could not be studied, eg. SSIIIa gene affects amylose content (Gao et al. 1998; Fujita et al. 2007; Li et al. 2011) and starch debranching enzymes (Kubo et al. 1999). Therefore, more research work is required to define the functions of other genes on starch property variation between indica and japonica rice.

\section{The relation between amylose content and starch}

\section{functional properties}

Starch AC has been reported to be negatively correlated with the gelatinization $\left({ }^{1} \mathrm{Tp}\right)$ and pasting properties (PV) of starches (Sasaki et al. 2000; Chen et al. 2003; Varavinit et al. 2003; Park et al. 2007). In the current study, among the six allele groups, the Wxi allele is determined as a major allele for AC increase. This result for $W x i$ allele is consistent with early works on $W x^{a}$, a high mRNA expressing $W x$ allele, which has been identified as a major gene controlling amylose content (Sano 1984; Wang et al. 1995). $W x^{a}$ allele explains most of the difference in increasing amylose content, decreasing the PV and BD of RVA in an indica rice variety, which greatly affects the quality of cooked rice (Umemoto et al. 2008). In addition, the current study also shows the impact of SSIi, SSIIaj and SBEIi alleles in determining difference of amylose content in RIL lines comparing to their corresponding allele pairs. The nature of high AC for Wxi allele group remains to be studied further. Analysis of a complete set of 64 potential combinations may explain the nature of its high AC. In the perspective of starch functional properties, high values were determined for paste and thermal properties in $W x j$ allele and other three relatively low amylose alleles (SSIj, SSIIai and $S B E I j$ ) compared to their counterpart-alleles. This correlation again confirms starch paste and thermal properties are negatively regulated by the ratio of amylose produced in the grains. 


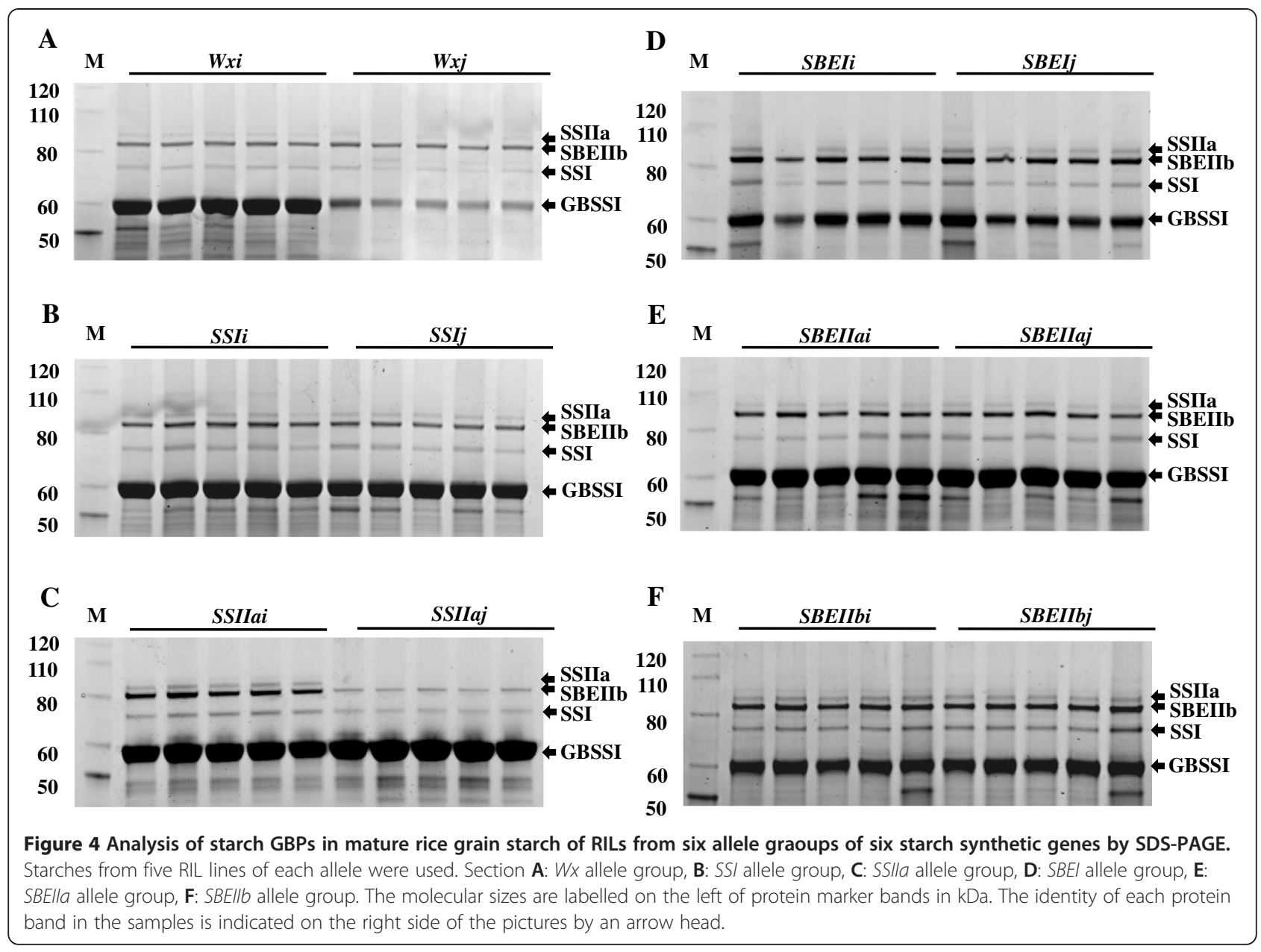

The relation between amylopectin chain length distribution and starch functional properties

Previous studies showed that short chains (DP $6 \sim 10$ ) and intermediate (DP $11 \sim 24$ ) chains of amylopectin are the major components of amylopectin structure in rice endosperms (Hizukuri 1985, 1986; Hizukuri et al. 1989). The variations in short and intermediate chains were related with starch pasting and thermal property changes (Park et al. 2007). Thus, it is interesting to investigate the impact of indica and japonica alleles on the relation

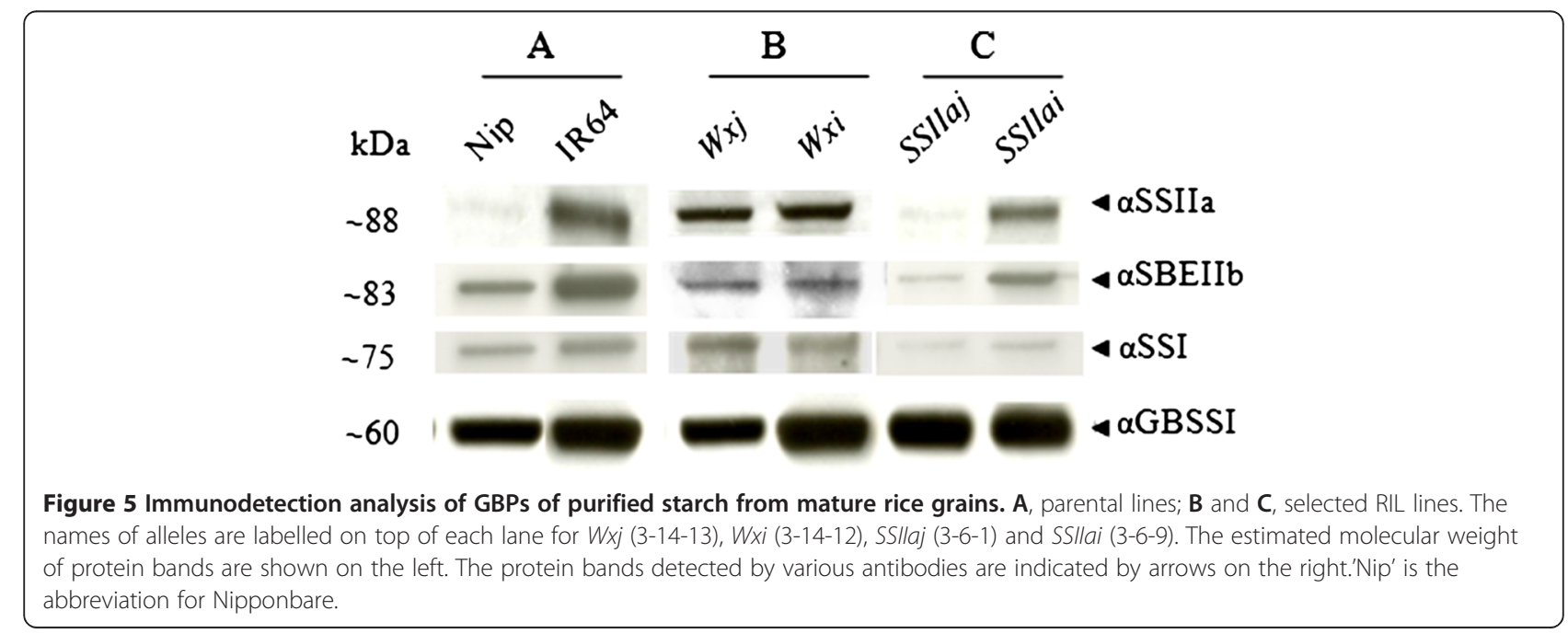


of amylopectin CLD and starch functional properties. Since $\mathrm{R}_{\mathrm{CL10} / 24}$ was the same between $W x i$ and $W x j$ alleles, it suggests Wx protein was not involved in the synthesis of short chains in amylopectin. The roles of SSIj allele in increasing starch $\mathrm{R}_{\mathrm{CL12/24}}$ value and starch properties in rice grain were reported by early researchers (Takemoto-Kuno et al. 2006) through comparing starches from progeny lines derived from the crossing population between indica and japaonica rice. However, such increasing starch $R_{\mathrm{CL10/24}}$ value was not observed from SSIj allele in this work although starch from SSIj allele contained more short chains less than DP 24 comparing to that from SSIi allele. The discrepancy between early publication (Takemoto-Kuno et al. 2006) and our current work may be due to the different varieties used to develop RIL population or the effects from SSIj and SBEIIbj combination. Umemoto et al. (2008) studied a near-isogenic line-NIL $\left(S S I^{k}\right)$ containing a $S S I$ allele from an indica rice on a japonica rice background and concluded the variation in SSI alleles between indica and japonica rice hardly affected starch peak viscosity and gelatinization temperatures. The SSIIaj allele starch showing more short chains and less intermediate chains was determined with a remarkably high $\mathrm{R}_{\mathrm{CL10/24}}$. This variation led to distinctive characteristics of starch properties with the lowest PV, BD, and lowest gelatinization temperatures. Despite other alleles within $W x i$ allele group showed lower PV and BD than IR64, they were comparable in $\mathrm{R}_{\mathrm{CL10/24}}$ values. Consequently, these alleles barely contribute to the variations of RVA and DSC characteristics within the Wxi allele group. Within the $W x j$ allele group, the SSIIai allele was prevalent showing similar CLD patterns, which was significantly different from Nipponbare. The SBEIIbj allele had a significantly higher $\mathrm{R}_{\mathrm{CL} 10 / 24}$ value comparing to SBEIIbi, but it was much lower than that of Nipponbare. The SBEIIbj allele had abundant short chains in amylopectin with lower $\mathrm{PV}, \mathrm{Tr}$ and lower FV and lower ${ }^{1} \mathrm{Te}$ than SBEIIbi allele.

Given all the analyses above, $\mathrm{R}_{\mathrm{CL10/24}}$ is negatively correlated with starch viscosity and thermal gelatinization properties. An increase of $\mathrm{R}_{\mathrm{CL10} / 24}$ can reduce the $\mathrm{PV}$ values of RVA and gelatinization temperature of DSC due to the increase of short chains proportion and/or decrease of intermediate chains proportion. SSIIaj is a major allele and SBEIIbj is a minor allele in the determination of $\mathrm{R}_{\mathrm{CL10/24}}$ in this study. Interestingly, starch PV and gelatinization temperatures of the SSIIaj allele are even lower than Nipponbare, though the SSIIaj allele $\mathrm{R}_{\mathrm{CL} 10 / 24}$ is smaller. This is because SSIIaj allele combines with Wxi allele in the corresponding RILs. Such changes of starch properties were also reported by Umemoto et al. (2008) through study of near-isogenic line-NIL (Wxa) which contained a chromosome segment containing a Wx allele from an indica rice (Kasalath) on a janonica (Nipponbare) rice background. Our analyses here demonstrate that both high amylose content and high $\mathrm{R}_{\mathrm{CL10/24}}$ regulate starch properties by lowering starch PV and gelatinization temperatures. SSIIaj and SBEIIbj alleles contribute to high $\mathrm{R}_{\mathrm{CL} 10 / 24}$. Therefore, the novel starch functional properties of SSIIaj allele is probably the result of the combination of major alleles, SSIIaj (high $\mathrm{R}_{\mathrm{CL10} / 24}$ ) and Wxi (high AC), with minor alleles, SBEIi and SBEIIbj.

\section{The relation between starch structure and starch granule bound proteins}

The $W x$ allele group showed significant variation in the abundance of GBSSI in the starch granule of rice grains. RILs with the $W x j$ allele contained a relatively low abundance of GBSSI and a low level of starch AC compared with those with the $W x i$ allele. This was consistent with previous studies which demonstrated that Wxj allele produces low levels of mature enzymes (Cai et al. 1998; Hirano et al. 1998), and rice varieties with $W x i$ and $W x j$ alleles have different levels of starch AC in rice grains (Sano 1984; Wang et al. 1995). However, the change of GBSSI abundance inside starch granules did not affect the abundance of other starch biosynthetic enzymes in starch granules. SSIIa was the other protein with varied abundance in starch granules between indica and japonica alleles. Moreover, SSIIaj allele starch contained a reduction of SSI and SBEIIb. According to Umemoto et al. (2004), the SSIIa protein from IR64 and Nipponbare differ by two amino acid substitutions, the protein from Nipponbare has reduced SSIIa activity. Furthermore, the reduced SSIIa activity resulted in the decrease of SSIIa abundance in starch granules, and ultimately produced amylopectin with increased $\mathrm{R}_{\mathrm{CL10/24}}$ values and slightly increased AC. Although SSIi, SBEIIaj and SBEIIbj alleles showed limited effects on starch structures and properties, the changes in the expression levels of these proteins were hardly detected from current study.

A recent study in rice and maize suggested that the majority of GBPs are involved in starch biosynthesis (Koziol et al. 2011). It indicated that the GBPs are important for the starch structure and properties. In our study, the remarkable changes in GBPs of SSIIa and Wx alleles observed in starch granules is associated with the pronounced changes in starch structure and functional properties as described earlier. This is consistent with the observations by numerous previous studies on single or double starch biosynthetic gene-recessive mutants of SSI, SSIIa and SBEIIb proteins, not only in rice but other cereals (Morell et al. 2003; Nakamura et al. 2005a; Kosar-Hashemi et al. 2007; Grimaud et al. 2008; Li et al. 2011). Therefore, detecting significant changes in the abundance and composition of GBPs is an alternative approach to screen variants with favorable starch structure and functional properties. 


\section{Conclusions}

This study showed that rice Wxi and SSIIaj alleles are major contributors, whereas SSIi, SBEIi, SBEIIai and $S B E I I b j$ alleles are minor contributors to rice starch properties between indica and japonica rice. The combination of SSIIaj and Wxi with SBEIIbj and SBEIi alleles has accumulative effects lowering PV and gelatinization temperature, which leads to the novel functional properties of SSIIaj allele starch in the study. Starch AC and the amylopectin CLD determine a number of starch functional properties. The $\mathrm{R}_{\mathrm{CL10} / 24}$ in amylopectin is negatively correlated with $\mathrm{PV}$ and $\mathrm{BD}$ of paste property and GTs of thermal property. Moreover, we suggest that the alteration of GBPs inside starch granules is a potential indicator for significant changes in starch structure and functional properties. A future study involving 64 possible combinations for the 6 gene groups in a common genetic background in rice will lead to an in-depth understanding of starch structures and functional properties.

\section{Methods \\ Plant materials}

Two rice (Oryza sativa) parental lines [cultivars IR64 (indica pollen donor) and Nipponbare (japonica acceptor)] were used for producing a RIL population. Four F2 seeds from one F1 seed were produced (kindly provided by Dr. Narayana Upadhyaya). Only one F2 plant was used for producing all RILs in this study. The detailed steps for selecting RILs for six allele groups are listed in Figure 1. All plants including RILs and their parental lines were grown in a glass house of a plant growth facility at CSIRO Agriculture Flagship (Canberra, ACT, Australia) at $27^{\circ} \mathrm{C}$ under natural light. Mature seeds were harvested for starch preparation, and starch structure and properties analysed.

\section{Genomic DNA preparation}

The genome DNA was extracted with a quick DNA extraction method. Fresh young leaves of $2 \mathrm{~cm}$ length were sampled into microfuge tubes on ice and immediately ground in $66 \mu \mathrm{l}$ of $0.5 \% \mathrm{NaOH}$ using a micropestle. Samples were then incubated in a boiling water bath for one minute, and diluted with $120 \mu \mathrm{l}$ of $0.1 \mathrm{M}$ Tris- $\mathrm{HCl}$, pH8.0. DNA was precipitated by adding $180 \mu \mathrm{l}$ isopropanol and $18 \mu \mathrm{l} 3 \mathrm{M}$ sodium acetate, pH5.2 to sample tubes and gently mixed by reversing the tube, and incubated at room temperature for $5 \mathrm{~min}$. The supernatant was discarded after the centrifugation for $30 \mathrm{~min}$ at $3000 \mathrm{~g}$, and the pellet was air dried at room temperature for $1 \mathrm{~min}$ and washed with $180 \mu \mathrm{l}$ of $70 \%$ $(\mathrm{V} / \mathrm{V})$ ethanol. After centrifugation at $3000 \mathrm{~g}$, the genomic DNA in the pellet was then dissolved in $50 \mu \mathrm{l}$ MilliQ water.

\section{Genotyping of selected genes}

The genotypes of RILs for the selected genes in the study were identified based on a PCR approach using published markers (Yan et al. 2007; Tian et al. 2010). For each allele, five individual plants were selected for generating data for statistical analysis. Due to the limitation of material availability, only three of five plants with more grains of each allele were used for starch property characterization. Alleles of indica derived from IR64 were assigned as Wxi, SSIi, SSIIai, SBEIi, SBEIIai and $S B E I I b i$, and corresponding japonica alleles derived from Nipponbare were named as Wxi, SSIj, SSIIaj, SBEIj, SBEIIaj and SBEIIbj. The alleles were segregated in different combinations during subculture, and F5 homozygotes were used for further analyses.

Three F3 RILs (3-5, 3-6 and 3-14) from one F2 line were used for the further selection of different combinations of the 6 genes by self-pollination due to their homozygosity and heterozygosity of six different genes in three F3 lines. Line 3-5 was homozygous for SSIi, SSIIai and SBEIIai and heterozygous at Wx, SBEI and SBEIIb loci. Among the F4 selfing progeny of F3 line 3-5, lines 3-5-1, 3-5-2 and 3-5-15 were homozygous for SBEIIbi and Wxj, SBEI $i$ and $W x j, S B E I i$ and $W x i$, respectively. They were further grown to obtain homozygous lines for indica and japonica type for SBEI and SBEIIb genes. In the F5 progeny, line 3-5-1-2 and line 3-5-1-8 were homozygous at SBEI, with SBEIi and SBEIj, respectively; line 3-5-2-1 and line 3-5-15-11 were homozygous at SBEIIb, with SBEIIbj; line 3-5-2-2 was homozygous for SBEIIbi. Line 3-6 was homozygous for Wxi, SSIj and SBEIi and heterozygous at SSIIa, SBEIIa and SBEIIb loci. Among the F4 progenies of line 3-6, three lines were homozygous for SSIIaj, SBEIIaj and SBEIIbj (line 3-6-1), SSIIai, SBEIIaj and SBEIIbj (line 3-6-9), and SSIIai, SBEIIai and SBEIIbj (line 3-6-20), respectively. RIL line 3-14 was homozygous at SSIi, SSIIai, SBEIi, SBEIIaj and SBEIIi, and heterozygous at Wx. Wxi and $W x j$ alleles were obtained in lines 3-14-12 and line 3-14-13 lines in the F4 progeny.

\section{Starch preparation}

Whole grains of each line (approximately $100 \sim 150 \mathrm{mg}$ ) were ground in a capsule with ball bearing using an ESPE CapMix $^{\text {тा }}$ (model $3 \mathrm{M}$, AU). Grinding was done for $30 \mathrm{sec}$ three times. The wholemeal flour was then washed with $0.005 \% \mathrm{NaOH}$ by vigorous vortexing for 2 minutes followed by filtration through $0.5 \mathrm{~mm}$ nylon sieves. Each sample was then washed three times by vortex mixing and centrifugation at $5000 \mathrm{~g}$ for $5 \mathrm{~min}$. The starch pellet was resuspended in a phosphate buffer $(50 \mathrm{mM}, \mathrm{pH} 7.5)$ containing proteinase $\mathrm{K}(50 \mu \mathrm{g} / \mathrm{ml})$ and incubated at $37^{\circ} \mathrm{C}$ for $2 \mathrm{hrs}$. After 5 min centrifugation at $5000 \mathrm{~g}$, the pellet was suspended in water and centrifuged. Thus water wash was repeated three 
times. The starch pellet was then washed with acetone, centrifuged and the residual starch was air dried at $37^{\circ} \mathrm{C}$ overnight.

\section{Amylose content assay}

The amylose content of samples was determined by analysing debranched starches using size-exclusion chromatography (SEC) with Ultrahydrogel (Waters, Milford, MA, USA) as described previously (Butardo et al. 2011). Pullulan standards (Shodex P-82) calibrated with the Mark-Houwink-Sakaruda equation were used for the estimation of the molecular weight from the elution time (Castro et al. 2005; Ward et al. 2006). Samples were prepared and analysed in triplicate.

\section{Chain length distribution of debranched starch}

Samples were prepared as previously described with modifications (O'Shea and Morell 1996). For each sample, rice wholemeal $(10 \mathrm{mg})$ was first presoaked in $40 \mu \mathrm{l}$ ethanol and then incubated with $200 \mu \mathrm{l} \mathrm{NaOH}(0.25 \mathrm{M})$ and $600 \mu \mathrm{l} \mathrm{MilliQ}$ water in a boiling water bath with stirring for $10 \mathrm{~min}$. The samples were then neutralized in the sodium acetate buffer $(0.2 \mathrm{M}$ sodium acetate and $3.6 \% \mathrm{~V} / \mathrm{V}$ glacial acetic acid). Afterwards, $10 \mu \mathrm{l}$ of isoamylase (1000 U/ml, Megazyme) was added to each sample to debranch the starch by incubating at $50^{\circ} \mathrm{C}$ for $2 \mathrm{~h}$. The samples were boiled again for $10 \mathrm{~min}$ to denature isoamylase and centrifuged at $15000 \mathrm{~g}$ for $10 \mathrm{~min}$. A $50 \mu \mathrm{l}$ aliquot of supernatant was taken and dried down in a speed vacuum. The dried samples were dissolved in APTS (8-Aminopyrene-1,3,6-trisulfonic acid trisodium salt, BECKMAN COULTER) sodium cyanoborohydride buffer (containing $5 \mathrm{mg}$ APTS labeling dye in $48 \mu \mathrm{l}$ 15\% acetic acid) and incubated overnight at $50^{\circ} \mathrm{C}$. After the incubation, the samples were boiled in a urea solution $(6 \mathrm{mM})$ for $1 \mathrm{~min}$ and filtered through Wizard miniColumns (Promega). Determination of the chain length distribution (CLD) of amylopectin was conducted by fluorescence-activated $\mathrm{CE}$ as previously described (O'Shea and Morell 1996).

\section{Starch paste viscosity}

The polished rice grains (about $5 \mathrm{~g}$ ) were ground into flour in a stainless steel capsule on a ball bearing machine (model MM300, MEP instruments Pty Ltd, NSW, AU) for starch property analysis. A Rapid Visco Analyzer (model RVA-4SA, Newport Scientific, Sydney, NSW, AU) was used to determine starch paste viscosity parameters of the selected RILs. Three grams of rice grain wholemeal for each of three plants of each RIL were used for analyses. The program setting for the RVA comprised the following stages: hold at $60^{\circ} \mathrm{C}$ for $2 \mathrm{~min}$, heat to $95^{\circ} \mathrm{C}$ over $6 \mathrm{~min}$, hold at $95^{\circ} \mathrm{C}$ for $4 \mathrm{~min}$, cool to $50^{\circ} \mathrm{C}$ over $4 \mathrm{~min}$, and hold at $50^{\circ} \mathrm{C}$ for $4 \mathrm{~min}$.
The software Thermocline (Newport Scientific PtyLtd, Warriewood, NSW, AU) was used for data collection and analysis.

\section{Differential scanning calorimetry}

A Differential Scanning Calorimeter (model Pyris 1DSC, Perkin Elmer, Norwalk, CT, US) was used to determine the calorimetry profiles of flour samples of 3 different plants with triplicate for each RIL. For each line, $60 \mathrm{mg}$ of flour was weighed and mixed with water at ratio of $1.76: 1$. Afterwards, 3 aliquots with $45 \mathrm{mg}$ of each prepared from the starch-water premixed product were transferred to DSC pans separately, and used as replicates. The pans were then sealed and placed on the bench at room temperature to equilibrate overnight. A heating rate of $10^{\circ} \mathrm{C}$ min was used to heat the samples at $30 \sim 130^{\circ} \mathrm{C}$. Data was analysed using the instrument software provided by the manufacturer.

\section{Preparation of granule bound proteins from mature grains}

The starch prepared from mature grains by the method described above was used for preparing GBPs of each line. Following the procedures by Rahman et al. (1995) with some modifications, $4 \mathrm{mg}$ of starch was boiled in a protein denaturing extraction buffer $(50 \mathrm{mM}$ Tris buffer, $\mathrm{pH}$ 6.8, 10\% glycerol, 5\% SDS, 5\% $\beta$-mercaptoethanol, and bromophenol blue) at a ratio of $15 \mu \mathrm{l} / \mathrm{mg}$ starch for $5 \sim 10 \mathrm{~min}$. After the centrifugation at $13000 \mathrm{~g}$ for $20 \mathrm{~min}$, the supernatant was ready for SDS-PAGE analysis.

\section{SDS-PAGE and gel staining}

Supernatants $(25 \mu \mathrm{l})$ containing GBPs of each sample together with $5 \mu \mathrm{l}$ of marker were loaded onto SDS-PAGE gels, respectively. SDS-PAGE was conducted in Nu-PAGE 4-12\% gradient gels (Invitrogen) via MOPS-Tris-SDS buffer system containing (50 mM MOPS, $50 \mathrm{mM}$ Tris, 0.1\% SDS and $1 \mathrm{mM}$ EDTA) and operated at $20 \mathrm{~mA}$ constant current per gel in an Xcell SureLock ${ }^{\mathrm{Tm}}$ Mini Cell (Invitrogen). After electrophoresis, gels were stained by Sypro Ruby stain (Bio-Rad) following the manufacturer's instructions, and then visualized under a UV transilluminator (Uvitec, UK). MagicMark ${ }^{\mathrm{Tm}}$ XP Western Standard protein ladders (Invitrogen) were used to estimate the molecular weight of protein bands.

\section{Statistical analyses}

Statistical analyses were performed using Genstat version 9. Analysis of variance was performed for starch AC, amylopectin structure, RVA and DSC characteristics to obtain the least significant differences at $p<0.01$, looking at variations among the RILs. 


\section{Competing interests}

The authors declare that they have no competing interests.

\section{Authors' contributions}

$J$ has carried out the genotyping of RILs, analysis of starch CLD, RVA and DSC, analysis and interpretation of data, and drafting the manuscript. SAJ made substantial contributions to conception and design, and revise the manuscript critically for important intellectual content. AM made substantial contributions to conception and design. MKM made substantial contributions to conception and design. ZL made substantial contributions to conception and design, analysis and interpretation of data, revising manuscript critically for important intellectual content. All authors read and approved the final manuscript.

\section{Acknowledgements}

Jixun Luo was supported by CSC (Chinese Scholarship Council) and Australian National University scholarships. This work was funded by CSIRO Food Future National Research Flagship. Authors would like to thank Oscar Larroque for SEC and CE analysis and data collection; Hong Wang and Min Huang for amylose content assay and plant genomic DNA extraction; Chris Konik-Rose and Jeni Pritchard for their assistance with RVA and DSC analysis. Authors would also thank Chris Konik-Rose and for her critical reading of the manuscript and Vito M. Butardo for his suggestions during the course of this research.

\section{Author details}

${ }^{1}$ CSIRO Agriculture Flagship, GPO Box 1600, Canberra ACT 2601, Australia.

${ }^{2}$ College of Medicine, Biology and Environment, Australian National University, Canberra ACT 0200, Australia. ${ }^{3}$ International Rice Research Institute, Maligaya, Muñoz, Nueva Ecija, Philippines.

\section{Received: 28 August 2014 Accepted: 28 January 2015} Published online: 04 March 2015

\section{References}

Bao JS, Corke H, Sun M (2006) Nucleotide diversity in starch synthase lla and validation of single nucleotide polymorphisms in relation to starch gelatinization temperature and other physicochemical properties in rice (Oryza sativa L.). Theor Appl Genet 113:1171-1183

Biliaderis CG, Page CM, Slade L, Sirett RR (1985) Thermal-behavior of amylose-lipid complexes. Carbohydr Polym 5:367-389

Butardo VM, Fitzgerald MA, Bird AR, Gidley MJ, Flanagan BM, Larroque O, Resurreccion AP, Laidlaw HK, Jobling SA, Morell MK, Rahman S (2011) Impact of down-regulation of starch branching enzyme $\mathrm{lb}$ in rice by artificial microRNA- and hairpin RNA-mediated RNA silencing. J Exp Bot 62:4927-4941

Cai XL, Wang ZY, Xing YY, Zhang JL, Hong MM (1998) Aberrant splicing of intron 1 leads to the heterogeneous 5' UTR and decreased expression of waxy gene in rice cultivars of intermediate amylose content. Plant J 14:459-465

Castro JV, Ward RM, Gilbert RG, Fitzgerald MA (2005) Measurement of the molecular weight distribution of debranched starch. Biomacromolecules 6:2260-2270

Chen JJ, Lai FV, Lil CY (2003) Effects of compositional and granular properties on the pasting viscosity of rice starch blends. Starch-Stärke 55:203-212

Commuri PD, Keeling PL (2001) Chain-length specificities of maize starch synthase enzyme: studies of glucan affinity and catalytic properties. Plant J 25:475-486

Cuevas RP, Daygon VD, Morell MK, Gilbert RG, Fitzgerald MA (2010) Using chain-length distributions to diagnose genetic diversity in starch biosynthesis. Carbohydr Polym 81:120-127

Cuevas RP, Fitzgerald MA (2012). Genetic diversity of rice grain quality. In: Caliskan M (ed) Genetic Diversity in Plants ISBN: 978-953-51-0185-7, InTech, Available from: http://www.intechopen.com/books/genetic-diversity-in-plants/genetics-of-grainquality.

Fedoroff N, Wessler S, Shure M (1983) Isolation of the transposable maize controlling elements Ac and Ds. Cell 35:235-242

Fujita N, Yoshida M, Asakura N, Ohdan T, Miyao A, Hirochika H, Nakamura Y (2006) Function and characterization of starch synthase I using mutants in rice. Plant Physiol 140:1070-1084

Fujita N, Yoshida M, Kondo T, Saito K, Utsumi Y, Tokunaga T, Nishi A, Satoh H, Park JH, Jane JL, Miyao A, Hirochika H, Nakamura Y (2007) Characterization of SSIIla deficient mutants of rice: the function of SSIIla and pleiotropic effects by SSIIla deficiency in the rice endosperm. Plant Physiol 144:2009-2023
Gao M, Fisher DK, Kim KN, Shannon JC, Guiltinan MJ (1996) Evolutionary conservation and expression patterns of maize starch branching enzyme I and Ilb genes suggests isoform specialization. Plant Mol Biol 30:1223-1232

Gao M, Wanat J, Stinard PS, James MG, Myers AM (1998) Characterization of dull1, a maize gene coding for a novel starch synthase. Plant Cell 10:399-412

Gao ZY, Zeng DL, Cheng FM, Tian ZX, Guo LB, Su Y, Yan MX, Jiang H, Dong GJ, Huang YC, Han B, Li JY, Qian Q (2011) ALK, the key gene for gelatinization temperature, is a modifier gene for gel consistency in rice. J Integrat Plant Biol 53:756-765

Govindaraj P, Vinod KK, Arumugachamy S, Maheswaran M (2009) Analysing genetic control of cooked grain traits and gelatinization temperature in a double haploid population of rice by quantitative trait loci mapping. Euphytica 166:165-176

Grimaud F, Rogniaux H, James MG, Myers AM, Planchot V (2008) Proteome and phosphoproteome analysis of starch granule-associated proteins from normal maize and mutants affected in starch biosynthesis. J Exp Bot 59:3395-3406

Han XZ, Hamaker BR (2001) Amylopectin fine structure and rice starch paste breakdown. J Cereal Sci 34:279-284

Han YP, Xu ML, Liu XY, Yan CJ, Korban SS, Chen XL, Gu MH (2004) Genes coding for starch branching enzymes are major contributors to starch viscosity characteristics in waxy rice (Oryza sativa L). Plant Sci 166:357-364

Hannah LC, James M (2008) The complexities of starch biosynthesis in cereal endosperms. Curr Opin Biotech 19:160-165

He P, Li SG, Qian Q, Ma YQ, Li JZ, Wang WM, Chen Y, Zhu LH (1999) Genetic analysis of rice grain quality. Theor Appl Genet 98:502-508

Hirano HY, Eiguchi M, Sano Y (1998) A single base change altered the regulation of the waxy gene at the posttranscriptional level during the domestication of rice. Mol Biol Evol 15:978-987

Hizukuri S (1985) Relationship between the distribution of the chain-length of amylopectin and the crystalline- structure of starch granules. Carbohydr Res 141:295-306

Hizukuri S (1986) Polymodal distribution of the chain lengths of amylopectins, and its significance. Carbohydr Res 147:342-347

Hizukuri S, Takeda Y, Maruta N, Juliano BO (1989) Molecular- structures of rice starch. Carbohydr Res 189:227-235

Kharabian-Masouleh A, Waters DL, Reinke RF, Henry RJ (2011) Discovery of polymorphisms in starch-related genes in rice germplasm by amplification of pooled DNA and deeply parallel sequencing. Plant Biotech J 9:1074-1085

Kharabian-Masouleh A, Waters DLE, Reinke RF, Ward R, Henry RJ (2012) SNP in starch biosynthesis genes associated with nutritional and functional properties of rice. Sci Rep-Uk 2:557-565

Kosar-Hashemi B, Li Z, Larroque O, Regina A, Yamamori M, Morell MK, Rahman S (2007) Multiple effects of the starch synthase II mutation in developing wheat endosperm. Funct Plant Biol 34:431-438

Koziol AG, Marquez BK, Huebsch MP, Smith JC, Altosaar I (2011) The starch granule associated proteomes of commercially purified starch reference materials from rice and maize. J Proteomics 75:993-1003

Kubo A, Fujita N, Harada K, Matsuda T, Satoh H, Nakamura Y (1999) The Starchdebranching enzymes isoamylase and pullulanase are both involved in amylopectin biosynthesis in rice endosperm. Plant Physiol 121:399-410

Lanceras JC, Huang ZL, Naivikul O, Vanavichit A, Ruanjaichon V, Tragoonrung S (2000) Mapping of genes for cooking and eating qualities in Thai Jasmine rice (KDML105). DNA Res 7:93-101

Li Z, Li D, Du X, Wang H, Larroque O, Jenkins CL, Jobling SA, Morell MK (2011) The barley amo1 locus is tightly linked to the starch synthase Illa gene and negatively regulates expression of granule-bound starch synthetic genes. J Exp Bot 62:5217-5231

Mizuno K, Kobayashi E, Tachibana M, Kawasaki T, Fujimura T, Funane K, Kobayashi M, Baba T (2001) Characterization of an isoform of rice starch branching enzyme, RBE4, in developing seeds. Plant Cell Physiol 42:349-357

Morell MK, Kosar-Hashemi B, Cmiel M, Samuel MS, Chandler P, Rahman S, Buleon A, Batey IL, Li Z (2003) Barley sex6 mutants lack starch synthase lla activity and contain a starch with novel properties. Plant J 34:172-184

Nakamura T, Shimbata T, Vrinten P, Saito M, Yonemaru J, Seto Y, Yasuda H (2005a) Mutations in wheat starch synthase II genes and PCR-based selection of a SGP-1 null line. Theor Appl Genet 111:1072-1079

Nakamura Y, Francisco PB, Hosaka Y, Sato A, Sawada T, Kubo A, Fujita N (2005b) Essential amino acids of starch synthase lla differentiate amylopectin structure and starch quality between japonica and indica rice varieties. Plant Mol Biol 58:213-227 
Nakamura Y, Sakurai A, Inaba Y, Kimura K, Iwasawa N, Nagamine T (2002) The fine structure of amylopectin in endosperm from Asian cultivated rice can be largely classified into two classes. Starch-Stärke 54:117-131

Nakamura Y, Utsumi Y, Sawada T, Aihara S, Utsumi C, Yoshida M, Kitamura S (2010) Characterization of the reactions of starch branching enzymes from rice endosperm. Plant Cell Physiol 51:776-794

Nelson OE, Rines HW (1962) The enzymatic deficiency in the waxy mutant of maize. Biochem Biophys Res Commun 9:297-300

Nishi A, Nakamura Y, Tanaka N, Satoh H (2001) Biochemical and genetic analysis of the effects of amylose-extender mutation in rice endosperm. Plant Physiol 127:459-472

O'Shea MG, Morell MK (1996) High resolution slab gel electrophoresis of 8-amino-1,3,6-pyrenetrisulfonic acid (APTS) tagged oligosaccharides using a DNA sequencer. Electrophoresis 17:681-686

Park IM, Lbanez AM, Zhong F, Shoemaker CF (2007) Gelatinization and pasting Properties of waxy and non-waxy rice starches. Starch-Stärke 59:388-396

Rahman S, Kosar-Hashemi B, Samuel M, Hill A, Abbott DC, Skerritt JH, Preiss J, Appels R, Morell M (1995) The major proteins of wheat endosperm starch granules. Aust J Plant Physiol 22:793-803

Sano $Y$ (1984) Differential regulation of waxy gene expression in rice endosperm. Theor Appl Genet 68:467-473

Sasaki T, Takeshi Y, Matsuki J (2000) Effect of amylose content on gelatinization, retrogradation, and pasting properties of starches from waxy and nonwaxy wheat and their F1 Seeds. Cereal Chem 77:58-63

Satoh H, Nishi A, Yamashita K, Takemoto Y, Tanaka Y, Hosaka Y, Sakurai A, Fujita N, Nakamura Y (2003) Starch- branching enzyme I- deficient mutation specifically affects the structure and properties of starch in rice endosperm. Plant Physiol 133:1111-1121

Shure M, Wessler S, Fedoroff N (1983) Molecular-identification and isolation of the waxy locus in maize. Cell 35:225-233

Septiningsih EM, Trijatmiko KR, Moeljopawiro S, McCouch S (2003) Identification of quantitative trait loci for grain quality in an advanced backcross population derived from the Oryza sativa variety IR64 and the wild relative O. rufipogon. Theor Appl Genet 107:1433-1441

Takemoto-Kuno Y, Suzuki K, Nakamura S, Satoh H, Ohtsubo K (2006) Soluble starch synthase I effects differences in amylopectin structure between indica and japonica rice varieties. J Agricult Food Chem 54:9234-9240

Tan Y, Corke H (2002) Factor analysis of physicochemical properties of 63 rice varieties. J Sci Food Agricul 82:745-752

Tetlow IJ, Morell MK, Emes MJ (2004) Recent developments in understanding the regulation of starch metabolism in higher plants. J Exp Bot 55:2131-2145

Tian ZX, Qian Q, Liu QQ, Yan MX, Liu XF, Yan CJ, Liu GF, Gao ZY, Tang SZ, Zeng DL, Wang YH, Yu JM, Gu MH, Li JY (2009) Allelic diversities in rice starch biosynthesis lead to a diverse array of rice eating and cooking qualities. Proc Natl Acad Sci U S A 106:21760-21765

Tian ZX, Yan CJ, Qian QA, Yan S, Xie HL, Wang F, Xu JF, Liu GF, Wang YH, Liu QQ, Tang SZ, Li JY, Gu MH (2010) Development of gene-tagged molecular markers for starch synthesis-related genes in rice. Chin Sci Bull 55:3768-3777

Toriyama K, Heong KL, Hardy B (2005) Rice is Life: Scientific perspectives for the 21st century. Proceedings of the World Rice Research Conference (Los Baños, International rice research institute)

Tsai CY (1974) Function of waxy locus in starch synthesis in maize endosperm. Biochem Genet 11:83-96

Umemoto T, Aoki N (2005) Single-nucleotide polymorphisms in rice starch synthase Ila that alter starch gelatinisation and starch association of the enzyme. Funct Plant Biol 32:763-768

Umemoto T, Aoki N, Lin HX, Nakamura Y, Inouchi N, Sato Y, Yano M, Hirabayashi H, Maruyama S (2004) Natural variation in rice starch synthase lla affects enzyme and starch properties. Funct Plant Biol 31:671-684

Umemoto T, Horibata T, Aoki N, Hiratsuka M, Yano M, Inouchi N (2008) Effects of variations in starch synthase on starch properties and eating quality of rice. Plant Prod Sci 11:472-480

Umemoto T, Terashima K (2002) Activity of granule-bound starch synthase is an important determinant of amylose content in rice endosperm. Funct Plant Biol 29:1121-1124

Varavinit S, Shobsngob S, Varanyanond W, Chinachoti P, Naivikul O (2003) Effect of amylose content on gelatinization, retrogradation and pasting properties of flours from different cultivars of Thai rice. Starch-Stärke 55:410-415

Wang ZY, Zheng FQ, Shen GZ, Gao JP, Snustad DP, Li MG, Zhang JL, Hong MM (1995) The amylose content in rice endosperm is related to the posttranscriptional regulation of the waxy gene. Plant J 7:613-622
Ward RM, Gao QY, de Bruyn H, Gilbert RG, Fitzgerald MA (2006) Improved methods for the structural analysis of the amylose-rich fraction from rice flour. Biomacromolecules 7:866-876

Xie DL, Wang ZY, Hong MM (1999) The evidence for the excision of Tourist-Os6 from the promoter region of the rice sbe1 gene. Acta Phytophysiol Sinica 25:274-280

Yan C, Tian S, Zhang Z, Han Y, Chen F, Li X, Gu M (2007) The source of genes related to rice grain starch synthesis among cultivated varieties and its contribution to quality. Agr Sci China 6:129-136

Yu G, Olsen KM, Schaal BA (2010) Association between nonsynonymous mutations of starch synthase lla and starch quality in rice (Oryza sativa). New phytol 189:593-601

Zhao K, Tung CW, Eizenga GC, Wright MH, Ali ML, Price AH, Norton GJ, Islam MR, Reynolds A, Mezey J, McClung BCD, McCouch SR (2011) Genome-wide association mapping reveals a rich genetic architecture of complex traits in Oryza sativa. Nat Commun 2:467-476

Zheng X, Wu JG, Lou XY, Shi CH (2007) The QTL analysis on maternal and endosperm genome and their environmental interactions for characters of cooking quality in rice (Oryza sativa L.). Theor Appl Genet 116:335-342

\section{Submit your manuscript to a SpringerOpen ${ }^{\circ}$ journal and benefit from:}

- Convenient online submission

- Rigorous peer review

- Immediate publication on acceptance

- Open access: articles freely available online

- High visibility within the field

- Retaining the copyright to your article

Submit your next manuscript at springeropen.com 